\title{
Ventral Hernia with Obstruction without Mention of Gangrene
}

National Cancer Institute

\section{Source}

National Cancer Institute. Ventral Hernia with Obstruction without Mention of Gangrene. NCI Thesaurus. Code C35642.

The protrusion of contents of the abdominal cavity through the abdominal wall, at the site of the linea alba, which results in obstruction, without mention of necrosis of the herniated contents. 